Tecnologia e Inovação

Tecnology and Innovation
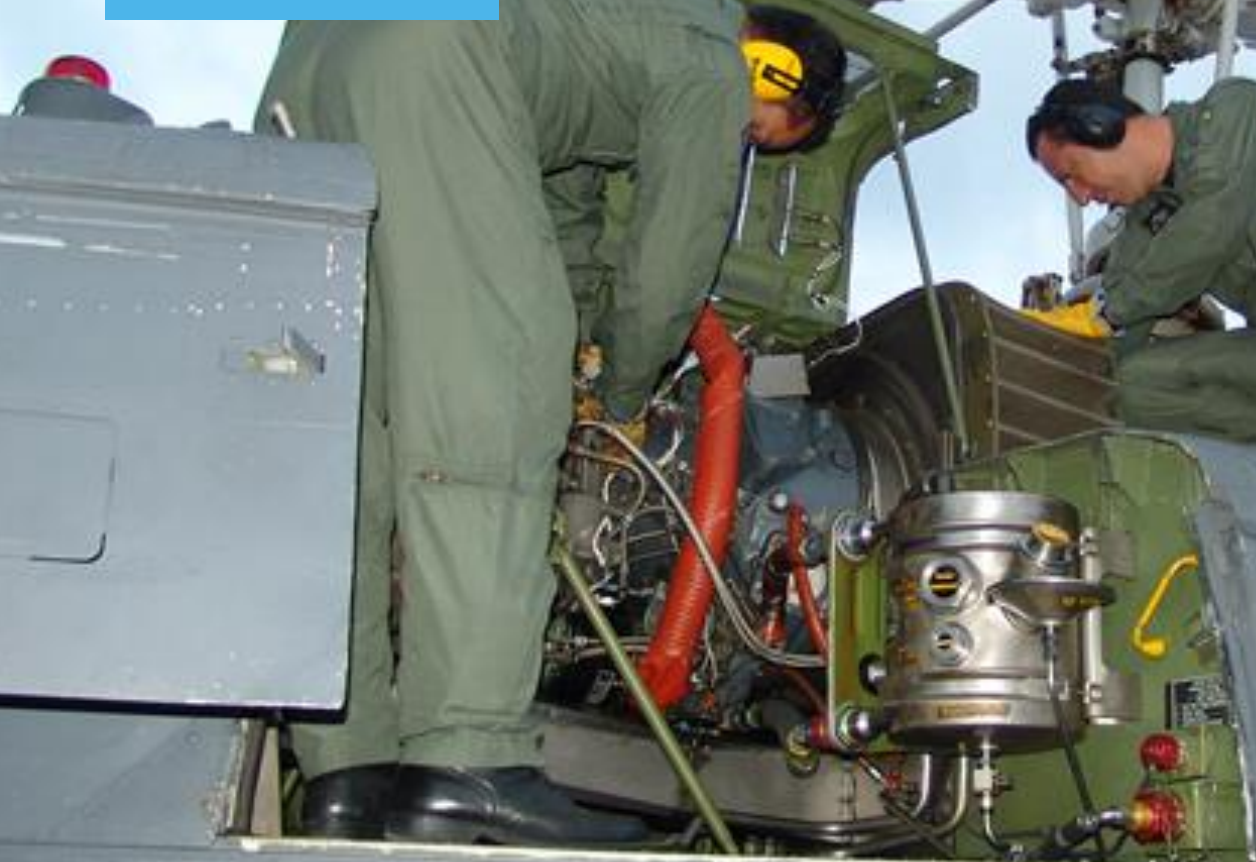

\title{
DISEÑO DE UN MÓDULO DE TRANSFORMACIÓN PARA SILLAS DE RUEDAS ELÉCTRICAS
} EN SILLA DE RUEDAS INTELIGENTE ${ }^{1}$.

DESENHO DE UM MÓDULO DE TRANSFORMAÇÃO PARA CADEIRAS DE RODAS ELÉTRICA INTELIGENTES².

Design Of A Transformation Module For Electric Wheelchairs In Smart Wheelchairs ${ }^{3}$.

Andrés Cifuentes, ${ }^{4}$ Mauricio Plaza, ${ }_{1}^{5}$ Santiago Rondón ${ }^{6}$

Universidad Militar Nueva Granada

CIENCIA Y PODER AÉREO

ISSN 1909-7050 / E- ISSN 2389-2468 / Volumen 11/ Enero-Diciembre de 2016/ Colombia/ Pp. 202-213

Recibido: 21/08/2016

Aprobado evaluador interno: 02/09/2016

Aprobado evaluador externo: 12/09/2016

Doi: http://dx.doi.org/10.18667/cienciaypoderaereo.532 
Para citar este artículo:

Cifuentes, A., Plaza, M., \& Rondón, S. (2016). Diseño de un módulo para transformar sillas de ruedas eléctricas en sillas de ruedas inteligentes. Ciencia y Poder Aéreo, 11 (1), 202-213.

Doi http://dx.doi.org/10.18667/cienciaypoderaereo.532.

${ }^{7}$ Artículo científico original derivado del proyecto IMPING-2135 titulado "Diseño de dispositivo exoesquelético mecánico para ayuda de personas con problemas de tensión muscular y actividades repetitivas de la Universidad Militar Nueva Granada (UMNG), Bogotá D.C., Colombia (2016).

${ }^{2}$ Artigo científico original derivado do projeto IMPING-2135 com título "Desenho de um dispositivo exoesquelético mecânico para ajuda de pessoas com problemas de tensão muscular e atividades repetitivas da Universidade Militar Nueva Granada (UMNG), Bogotá D.C., Colômbia (2016).

${ }^{3}$ Original scientific article derived from the IMPING-2135 project titled "Design of mechanical exoeskeletal device to help people with muscular tension problems and repetitive activities of the Nueva Granada Military University (UMNG), Bogota D.C., Colombia (2016).

${ }^{4}$ Ingeniero Mecatrónico de la Universidad Nacional de Colombia, Magíster en Ingeniería Mecatrónica de la Universidad Militar Nueva Granada. correo electrónico: andres.cifuentes@unimilitar.edu.co

${ }^{5}$ Ingeniero Eléctrico Universidad de los Andes, Doctor en Ingeniería con énfasis en Realidad Virtual y Bioingeniería. correo electrónico: mauricio.plaza@ unimilitaredu.co

${ }^{6}$ Estudiante de Ingeniería Mecatrónica de la Universidad Militar Nueva Granada. correo electrónico: u1802364@unimilitar.edu.co
Resumen: En el presente artículo se describe el proceso de diseño y validación de un sistema embebido adaptable a una silla de ruedas eléctrica estándar para su trasformación en una silla de ruedas inteligente, entendiendo a esta última, como un sistema integrado completo, que permite al usuario de la silla de ruedas moverse de la misma forma que suele hacerlo por medio de los controles clásicos tipo palanca de una silla de ruedas eléctrica comercial, y que además preste algunas funciones en línea como el monitoreo de funciones vitales y movimiento semiautomático de la silla de ruedas en un ambiente conocido, utilizando una interfaz gráfica y un software para planeación de trayectorias.

Palabras clave: señales biométricas; sistemas embebidos; sistemas inteligentes; silla de ruedas inteligente.

Resumo: No presente artigo é descrito o processo de desenho e validação dum sistema absorto, adaptável a uma cadeira de rodas elétrica normal para sua transformação numa cadeira de rodas inteligente, entendendo essa ultima, como um sistema integrado completo que ajuda ao usuário da cadeira de rodas se movimentar do mesmo jeito que ele costuma faze-lo por meio dos controles clássicos tipo palanca duma cadeira de rodas elétrica comercial e que ainda tenhas algumas funções mais online como o monitoramento de funções vitais e movimento semiautomático da cadeira de rodas num ambiente conhecido, utilizando uma interfase gráfica e um software para o planejamento de trajetórias.

Palavras-chave: sinais biométricos; sistemas absortos; sistemas inteligentes; cadeira de rodas inteligente.

Abstract: This article describes the process of design and validation of an embedded system adaptable to a standard electric wheelchair for its transformation to a smart wheelchair, the latter being understood as a complete integrated system which allows the wheelchair user to move in the same way as he usually does through the classic levers controls of a commercial electric wheelchair and that, in addition, provides some online functions such as monitoring vital functions and semiautomatic movement of the wheelchair in a known environment, using a graphic interface and a software for planning the trajectories.

Key Words: Biometric Signals; Embedded Systems; Intelligent Systems; Smart Wheelchair. 


\section{Introducción}

En la mayoría de los países a nivel mundial, la esperanza de vida ha aumentado de forma significativa, gracias a los avances en las ciencias médicas, sin embargo, esto conlleva una serie problemas relacionados con el envejecimiento de la población, como dolencias derivadas del desgaste de los tejidos y problemas de salud propios de la tercera edad. De hecho, según expertos convocados en el International Day of Disabled Persons (ILO, 2007), para el año 2025 el 20\% de la población tendrá algún tipo de discapacidad o requerirá asistencia pre ambulatoria de forma regular (Noda, Kawaguchi, \& Terashima, 2010).

Tal aumento en la cantidad de personas que requieren ayuda médica de tipo asistencial, ha comenzado a preocupar gobiernos de diversos países como Japón y Canadá, en donde se espera que en menos de 15 años el porcentaje de la población que pertenezca al grupo de adultos mayores cambie del $23.1 \%$ al $25.6 \%$ en el primero, y de $12.9 \%$ al $22.5 \%$ en el segundo. (Ministerio de Asuntos Internos y Comunicaciones Oficina de Estadísticas, 1996) (Panam Post, 2015) (Statistics Canada, 2015).

Las muletas y sillas de ruedas son los elementos de apoyo médico más empleados en los casos de enfermedades degenerativas que limitan la movilidad de los sistemas musculo-esquelético en adultos. En la actualidad, una amplia gama de sillas de ruedas suelen incluir sistemas electromecánicos para facilitar la función de desplazamiento, algunas de ellas incluyen sistemas sofisticados que diversifican sus funciones, éstas últimas son llamadas sillas de ruedas inteligentes. Una silla de ruedas inteligente, en adición a la capacidad motriz que comparte con las sillas de ruedas eléctricas, cuenta con mecanismos de medición de variables vitales, sistemas de comunicación y transmisión de información, mecanismos de monitoreo, adaptación a distintos comandos de control diferentes a la palanca de mando e incluso capacidades de navegación autónoma o semiautónoma (AL-Rousan \& Assaleh, 2011).

Los primeros desarrollos en el área enfocaron sus esfuerzos en el problema básico de traslación sin choque con elementos del entorno, normalmente conocido como generación de trayectorias con evasión de obstáculos (AL-Rousan \& Assaleh, 2011). Con el pasar de los años, se hizo evidente la necesidad de crear sistemas más sofisticados centrados en el usuario, entonces, el problema dejó de ser solamente permitir el desplazamiento de un paciente en silla de ruedas sin necesidad de ayuda humana adicional, sino que dicho traslado fuera seguro y no violento, al mismo tiempo que se logra permitir el acceso a mecanismos adicionales para que el paciente aumente el rango de interacción con su entorno y su nivel de independencia.

A pesar de los esfuerzos de la comunidad investigativa, la comercialización de estas soluciones de ingeniería para sillas de ruedas inteligentes no ha sido difundida. La mayoría de la población que requiere una silla de ruedas en los países desarrollados utiliza sillas eléctricas comerciales y se desconoce que un sistema de adaptación podría ser más exitoso que un desarrollo completo de una silla de ruedas inteligente.

Aprovechando que la movilidad para este tipo de sillas de ruedas se logra empleando motores eléctricos, es posible implementar sistemas de control electrónico que gobiernen las acciones de movimiento de la silla completa, en cuyo caso deben considerarse las limitantes en las señales de control, para no aumentar significativamente los consumos de energía, ni comprometer la integridad del sistema (Noda, Kawaguchi, \& Terashima, 2010).

En adición a lo expuesto, existe un interés particular en realizar mejoras y nuevas adaptaciones a los elementos de apoyo médico asistencial relacionados con dolencias que en la tercera edad se vuelven crónicas. En el presente artículo se propone una solución funcional a los problemas más presentados en dicha población, dificultades que incluyen, entre otras (Noda, Kawaguchi, \& Terashima, 2010):

- Limitación de movilidad y poco control autónomo.

- Necesidad de asistencia externa en los casos de perdida de movilidad marcada, para el manejo de elementos de interacción cotidiana como luces y puertas.

- Limitación de las actividades sociales a causa de las dolencias del paciente.

- Falta de monitoreo de las señales vitales en las personas con padecimientos que lo requieran.

Además, este documento presenta una revisión teórica y metodológica de los trabajos previos similares en el área, así como la descripción del proceso de diseño del módulo de transformación propuesto; una serie de pruebas de validación y verificación de funcionamiento se describe a continuación, seguida de un análisis concreto de los hallazgos obtenidos, que sustentan el apartado de conclusiones y de trabajos futuros presentados al final de este artículo. 


\section{Teoría del dominio y trabajos previos}

Las sillas de ruedas eléctricas son una solución ampliamente aceptada en los países desarrollados para la limitación de movimiento de los miembros inferiores. En el mercado se clasifican como "transportables", porque son de poco peso y fácil ensamble, de "poder" (powerbase), con unas especificaciones más robustas, de mayor tamaño e independencia de batería y "de trabajo pesado". Su función principal es el desplazamiento de pacientes en grandes áreas abiertas, lo que requiere de mayor capacidad de energía y más velocidad de desplazamiento.

La fuente de energía típica para los tipos de sillas de ruedas descritos son baterías de gran densidad de carga, entre las que encontramos baterías wet (liquid electrolyte), gel (or pasty -moisture electrolyte), o AGM (Absorbed Glass Mat), encargadas de alimentar motores eléctricos que usualmente pueden requerir hasta 5 amperios de corriente para la generación del torque requerido (Noda, Kawaguchi, \& Terashima, 2010).

Este tipo de sillas ha evolucionado significativamente, pero muchas personas que tienen problemas motrices son incapaces de accionar de forma efectiva los mecanismos de control por medio de un joystick tradicional, de hecho, en el hospital Hines VA se encontró, en colaboración con la facultad de ingeniería Aeroespacial y Mecánica de la universidad de Notre Dame, que de un $9 \%$ a $10 \%$ de las personas con discapacidad motriz severa, son incapaces de manipular de forma adecuada la silla de ruedas, o de mantener las acciones de control por periodos de tiempo prolongados, haciendo de los mecanismos de guiado autónomo una opción inviable para esta clase de pacientes (Fehr, Langbein, \& Skaar, 2000).

Diversas modificaciones y periféricos se han desarrollado con el fin de ampliar la versatilidad de la silla de ruedas eléctrica tradicional. Esta nueva generación de dispositivos médicos ha empezado a conocerse como "Smart wheelchairs" (sillas de ruedas inteligentes) y tienen el propósito de suplir diferentes necesidades en la interfaz hombre-máquina, de pacientes que requieren de una silla de ruedas y sufren limitaciones de movilidad en miembros superiores. Estas necesidades se derivan de la naturaleza de la enfermedad o discapacidad que el paciente sufra. Para 2012 se han perfeccionado dispositivos que pueden ser controlados con la barbilla o por medio de presión, soplando y succionando, que han sido pensados para las personas con amputación a nivel de brazo o antebrazo; lo que permite al paciente realizar acciones de control de accionamiento de tipo on-off, permitiéndoles manipular el arranque y frenado de la silla de ruedas, siempre y cuando ninguna habilidad cognitiva se encuentre afectada.

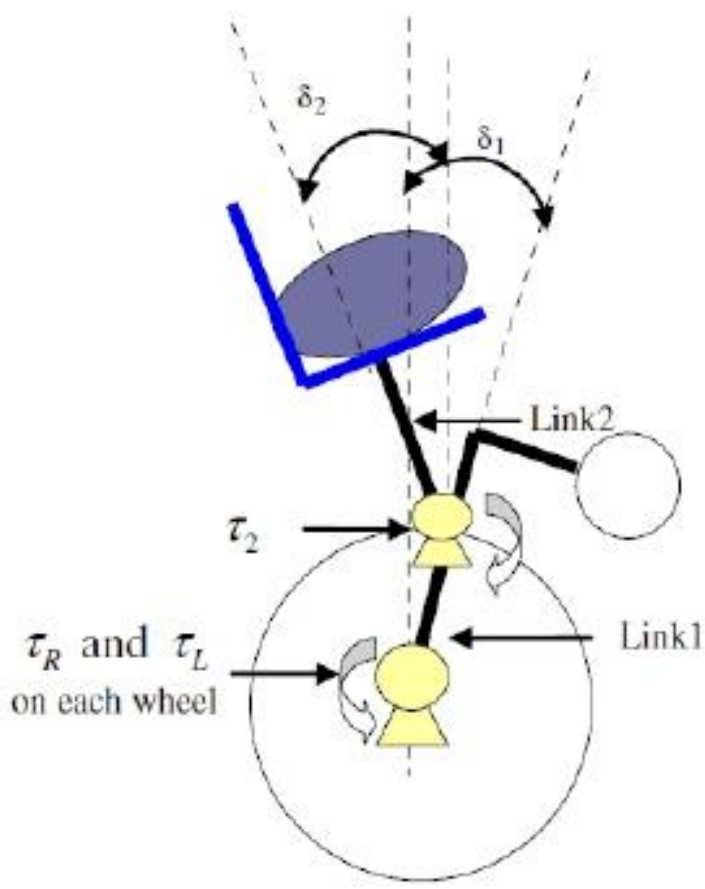

Figura 1. Modelo de silla de ruedas como péndulo doble invertido. Fuente: Ahmad, S., Siddique, N. H., \& Tokhi, M. O. (2011). A Modular Fuzzy Control Approach for TwoWheeled Wheelchair. Intell Robot Syst.

Esto demuestra que, parte de las tareas básicas que se desean realizar de forma simple e incluso autónoma por parte del dispositivo inteligente, se encuentran el desplazamiento de un punto a otro evadiendo obstáculos. Las versiones capaces de realizar esta tarea de forma automática, incluyen módulos de hardware y software que gobiernan la dinámica de los motores de la silla eléctrica empleando algoritmos como el método de los campos potenciales, el método VFH (Vector Field Histogram), o heurísticas que usan directamente la distancia entre la silla y los obstáculos para la generación de una trayectoria segura y los sistemas de control de seguimiento de ésta (Leishman, Horn, \& Bourhis, 2010).

Las últimas referencias en diseños y desarrollos de periféricos para esta clase de sillas incluyen el uso de sistemas de inteligencia artificial, técnicas híbridas de control de posición y movimiento, mejoramiento en las interfaces de usuario, reconocimiento de imágenes y voz, entre otros (Noda, Kawaguchi, \& Terashima, 2010).

Algunos diseños van incluso un poco más allá, y parten desde el diseño conceptual de un mecanismo para una silla de ruedas que permita cambiar su configuración física, como ejemplo tenemos el resultado de investigación de un equipo de investigadores en Malasia, que logró el desa- 
rrollo del modelo computacional de una silla de ruedas de dos ruedas similar a un doble péndulo invertido (Figura 1), el cual es un sistema altamente no lineal. El propósito de este tipo de diseño es permitir que el usuario alcance alturas más elevadas de las que lograría en una silla convencional; el control para este diseño fue un modelo neuro-difuso (Ahmad, Siddique, \& Tokhi, 2011).

En este desarrollo, el sistema puede cambiar de 4 a 2 ruedas de apoyo, lo que permite alcanzar mayor altura y realizar tareas complicadas como subir escaleras. Para lograrlo se implementaron controladores lógico-difusos que, una vez han estabilizado a la silla con el usuario en posición vertical, permiten el avance de la misma si el paciente así lo desea. Dos motores se emplean como actuadores independientes para cada una de las ruedas y un tercer motor se usa para lograr la verticalidad del usuario de la silla, las salidas de control fueron los torques en cada uno de los actuadores (Ahmad, Siddique, \& Tokhi, 2011).

En la mayoría de los casos mencionados, los sistemas aumentan el nivel de complejidad de la silla eléctrica y requieren de procesos específicos de manufactura. Así, el proceso de diseño de un módulo de adaptación en lugar de un rediseño total de la estructura de la silla eléctrica, se convierte en una opción razonable en situaciones en las que se busca una masificación para esta clase de dispositivos.

\section{Método}

El sistema fue diseñado para la transformación de diversos tipos de sillas de ruedas eléctricas de potencia media; el mismo se encuentra protegido por una carcasa plástica que permite la fácil visualización de los testigos de funcionamiento, cambios en la velocidad de titileo que indican funciones distintas y permiten a un usuario externo verificar la alimentación en el circuito y el funcionamiento de los módulos de captura y comunicación. En la Figura 2 se presenta la apariencia final del dispositivo diseñado.

La tarjeta principal del sistema cuenta con pines de conexión para módulos externos que incluyen los pines de adquisición de señales biométricas, pines de comunicación con las interfaces de potencia y pines de entrada para sensórica domótica adicional.

Se presenta en la Figura 3, la ubicación de los elementos de la tarjeta principal, cada una de las conexiones y la distribución de los pines para los periféricos fueron agrupados por simplicidad; la nomenclatura de cada una de las secciones de la tarjeta principal se muestra a continuación:

- A. Mecanismo de Reinicio.

- B. Encoders 1 y 2

- C. Sensor de pulso cardíaco.

- D. Pulsador de Parada de Emergencia.

- E. Entradas de sensórica adicional

- F. Led de funcionamiento de la brújula.

- G. Conector para la entrada de la Brújula electrónica

- H. Conector para datos del Joystick

- I. Selector de modo Automático/Manual

- J. Conector para la tarjeta de potencia y alimentación

- K. Conector para el sensor de presión diferencial (para la medición de la frecuencia respiratoria)

- L. Pulsador para calibración de la Brújula

- M. Módulo de comunicación inalámbrica (protocolo zigbee).

- N.Unidad de procesamiento y control.

- O. Led de funcionamiento del núcleo de procesamiento.

\section{Diseño detallado}

Las secciones pueden agruparse por su funcionalidad en el sistema, a continuación, presentaremos con más detalle cada uno de los módulos funcionales.

Adquisición y procesamiento de señales biométricas (secciones $C, K, E y N$ )

Esta parte agrupa los buses de datos y los conectores a los módulos de sensórica externos, el sistema está diseñado para recibir hasta 4 señales de sensórica diferentes, por defecto se implementaron señales de pulsioximetría y presión diferencial para frecuencia respiratoria, pero el módulo permite cambiar las señales monitoreadas o incluir más.

La adecuación de la señal debe mapear cualquier señal fisiológica a medir, de modo que su rango de voltaje no sea inferior a los 0 voltios DC ni superior a los 5 Voltios DC.

Debido a que las señales fisiológicas suelen tener frecuencias significativamente inferiores a las frecuencias de comunicación y procesamiento digital, se estableció una frecuencia máxima para las señales de entrada a este módulo de $50 \mathrm{KHz}$, correspondiente al $1 \%$ de la frecuencia del sistema. Esto garantiza un muestreo no inferior a 10 puntos por ciclo. Las operaciones de muestreo usan un máximo de $10 \%$ del tiempo empleado por el sistema para 


\section{Tapa superior en}

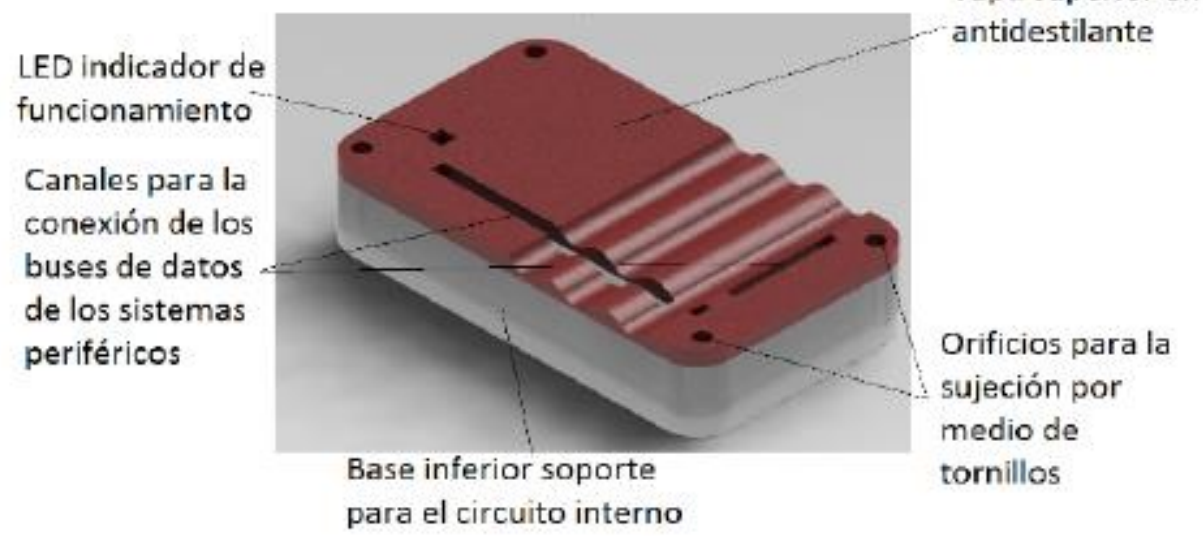

Figura 2. Render de la carcasa del embebido.

Fuente: elaboración propia.

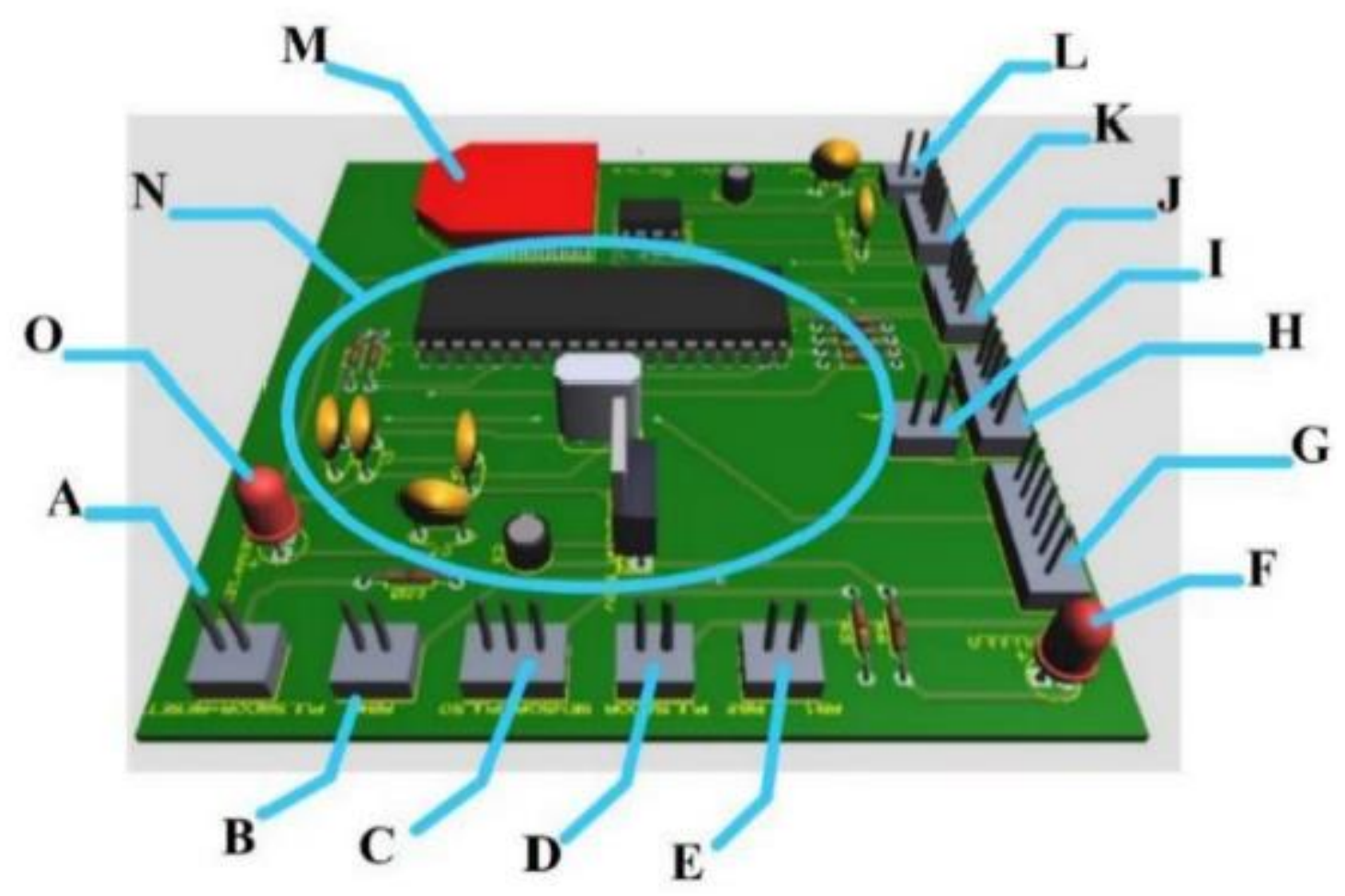

Figura 3. Diagrama de la tarjeta principal con etiquetas de cada una de las secciones. Fuente: elaboración de los autores. 
la elaboración de todas sus operaciones, lo que permite que cerca del $70 \%$ del tiempo de procesamiento, se dedique a las tareas de cálculo del controlador y operaciones de comunicación.

El módulo central requiere señales pre procesadas; cualquier filtro requerido en la señal ha de ser implementado en los periféricos de adquisición. El procesamiento digital de las señales vitales se logra por medio de un conversor análogo digital de 10 bits de resolución. Los procesos adicionales pueden ser añadidos, dependiendo del tipo de señal.

Módulo de acceso a periféricos y obtención de las señales de control (Secciones $A, B, D, G, H, I, J, L y N$ )

Este módulo agrupa las conexiones necesarias para la entrega de las señales de control a las etapas de potencia, la ubicación del bus de transmisión es cercano al módulo de procesamiento para minimizar el efecto de las capacitancias e inductancias parásitas en la señal de control. El sistema cuenta con una brújula magnética que indica la posición de la tarjeta con respecto al norte, esta información es empleada por el módulo para la orientación de la silla de ruedas.

El módulo de procesamiento central se basa en un microcontrolador PIC 18F4550, el cual cuenta con canales de conversión análogo digital con 10 bits de resolución, y puertos de entrada y salida digital de 8 bits de resolución.

\section{Módulo de comunicación y visualización (Secciones $F_{1}$ M, NyO)}

La unidad de procesamiento opera a una velocidad no inferior a 5MIPS, el hardware desarrollado es capaz de realizar todas las operaciones requeridas en un tiempo no superior a 10 ms, lo que implica que 100 muestras (en el peor de los casos) serán tomadas para cada una de las señales fisiológicas en cada segundo, nótese que este cálculo supera al requerimiento impuesto a las señales de monitoreo vital,discutidas en la sección del módulo de adquisición y monitoreo de señales biométricas.

Las señales son procesadas primero por el módulo interno de conversión análogo digital, toda la información requerida es empaquetada en una trama propia, que define una modificación al protocolo de comunicación serial rs232 (Microchip, 2006), que garantiza la sincronización adecuada entre los datos del sistema local, con el computador que contiene la interfaz gráfica de visualización.

\section{Descripción del funcionamiento interno del sistema}

De manera general, el sistema recibe una serie de datos entregados por los sensores y detecta si alguno está reportando una falla que pueda interrumpir el movimiento, como para emergencias, taquicardia, arritmia, fiebre, hipotermia o paro respiratorio. Si no detecta ninguna falla, el sistema procede a comparar la posición actual con la ingresada por el usuario; a continuación, el sistema procesa y envía la información necesaria tanto a los motores como a la interfaz gráfica de usuario.

El sistema envía un dato de sincronización, que será empleado periódicamente en el sistema computacional, de esta forma se garantiza la calibración de los datos y se minimiza la pérdida de información que puede ocurrir de manera intrínseca en el sistema de comunicación; los datos de los sensores y la frecuencia cardíaca son enviados a continuación, seguidos de un dato compuesto, que será interpretado por el programa en el equipo para calcular la orientación con respecto al norte de la silla de ruedas. Finalmente, un dato de control diferente es enviado al final de la trama; el dato es de vital importancia porque en este último byte se envía la información correspondiente a la dirección deseada de movimiento, en caso que el sistema se encuentre en modo manual, o la indicación al sistema computacional de entrar esté en modo automático, la forma general de la trama de datos se muestra en la Figura 4.

El sistema envía un dato de control al comienzo de la trama, esto garantiza una sincronización de los datos, el dato de control es generado por nuestro sistema de transformación, de manera que cualquier programa externo que desee leer la información de nuestro sistema, primero debe asegurarse de recibir el dato de control correcto, se recomienda que este dato sea leído de manera frecuente, con el fin de verificar errores de transmisión. A continuación, el sistema envía la información codificada en 10 bits, de los resultados de la conversión $A D C$ de las tarjetas de adquisición externa, resolución consistente con los módulos internos de conversión del microcontrolador; por defecto, el primer sensor corresponde a pulso cardíaco, la variable básica de condición de salud.

Luego del envío de los datos de los 4 sensores, un dato de 16 bits de resolución es enviado, este dato corresponde a los grados con respecto al norte de una brújula electrónica, con una resolución de 2 décimas de grado, la brújula tiene 3 modos de funcionamiento, comunicación serial por modo I2C, comunicación serial por protocolo RS232, o PWM; por defecto, el sistema trabaja con esta última opción, la brújula elegida es la CMPS10, el módulo de transformación incluye la brújula en cuestión dentro de su tarjeta madre, de modo que nuestro sistema es capaz de entregar la orientación de la silla de ruedas, in- 


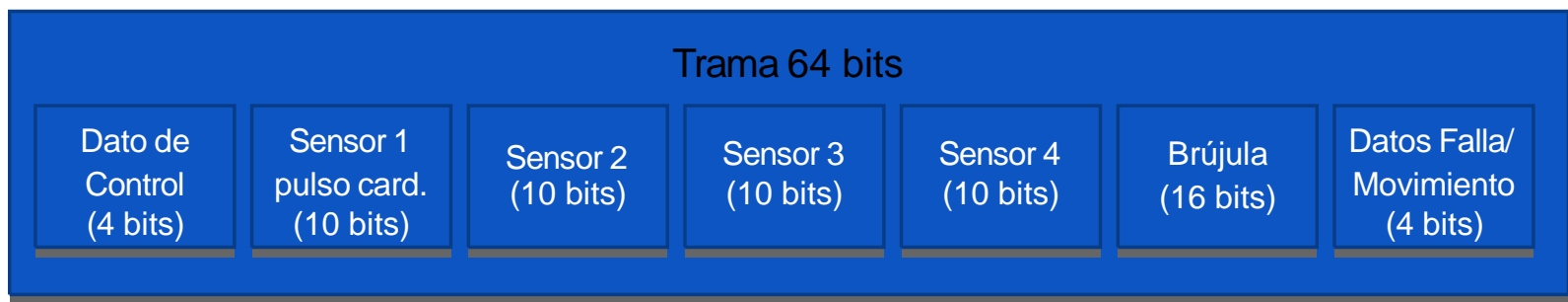

Figura 4. Trama de datos entregada por el sistema, para comunicar la tarjeta central con un PC Fuente: elaboración propia.

formación que puede ser empleada por un algoritmo de control de seguimiento de trayectorias.

Finalmente, un dato de 4 bits de longitud cierra la trama, en el mismo; valores de 0 a 15 son empleados para indicar la dirección de movimiento actual de la silla de ruedas (valores $0=$ quieto, $1=$ adelante, $2=$ atrás, $3=$ giro a la izquierda, 4= giro a la derecha), fallas programables (5 a 15) como bajo pulso cardíaco, taquicardia, obstrucción en las ruedas, entre otros. Un total de 10 fallas pueden ser programadas según la necesidad del usuario.

El sistema cuenta con 3 modos de funcionamiento: configuración, manual y automático.

En el modo configuración se llevan a cabo todas las configuraciones necesarias del sistema, la cantidad de sensores empleados se indica por medio de una interfaz gráfica de usuario, la velocidad de transmisión de datos, el tipo de sensor a emplear en cada uno de los puertos, lo que permite modificar la frecuencia de muestreo para cada uno (entre $50 \mathrm{KHz}$ y $1 \mathrm{~Hz}$.), y la habilitación del módulo de brújula electrónica, que en caso de no requerirse puede ser desconectado para ahorrar batería.

En el modo manual, el sistema entrega señales de control a los motores para mover la silla en la dirección que se desee y es determinada por una palanca de acción (joystick), de la misma forma que en una silla de ruedas eléctrica convencional, con esto se garantiza que nuestro módulo en ningún momento reducirá las capacidades que tenía la silla, antes de la implementación del módulo de transformación diseñado.

En el modo automático, el sistema recibe señales de control de un generador de trayectorias cualquiera. Las señales recibidas son adecuadas y enviadas a los motores. Los encoders adaptados a los motores, y el sistema de brújula electrónica entregan información al sistema que sirve como realimentación al sistema de control.

En cualquiera de los últimos dos casos, el usuario tiene la posibilidad de interrumpir el movimiento y frenar en seco por medio de un mecanismo de parada de emergencia, un pulsador de enclavamiento, cuya señal es prioritaria para el sistema, permite acciones de seguridad inmediatas en el módulo. La información de los encoders, los selectores de modo de movimiento y la brújula electrónica es enviada a un PC donde puede ser visualizada y se emplea en el cálculo de las referencias para los motores.

El sistema fue modelado como un robot diferencial, de modo que la dirección de la silla estará dada por la diferencia de velocidades entre las dos ruedas de la silla, para ello fue necesario desenvolver un sistema de control independiente para cada motor.

Tanto en el modo manual como en el automático el sistema lee, procesa y envía las señales biométricas del usuario a una interfaz gráfica, y en adición permite el movimiento de la silla.

Los mecanismos de asignación de los recursos de máquina debieron implementarse, para garantizar el funcionamiento de los sub sistemas de adquisición y procesamiento de las señales vitales, las tareas de comunicación con el módulo de visualización computacional, sistemas de monitoreo de los módulos de sensórica externa no relacionada con los mecanismos de captura biométrica, y el control de los sistemas actuados en la silla de ruedas y los periféricos del sistema domótico son coordinados por un módulo central. Las unidades de compensación se programaron para controlar el acceso al núcleo de procesamiento central de todos los subsistemas.

\section{Sintonización de los parámetros específicos de cada silla}

Para que el módulo se puede adaptar a cualquier silla de ruedas es necesario caracterizar los motores y generar un controlador adecuado para los mismos. Para ello se emplearon las reglas de sintonización de Ziegler-Nichols, según las cuales es posible diseñar un controlador eficiente para sistemas de primer orden. En el proceso de sintonización, el sistema envía una señal de control sostenida de valor constante y por medio de una captura en los motores de los encoders, se obtiene la respuesta del sistema en fun- 
ción del tiempo y para calcular las constantes que proveerán valores para un modelo generalizado de PID.

El sistema de transformación entrega un valor de voltaje constante de 12, 24 o 36 voltios, configurables por hardware, los datos de la lectura de los encoders son tomados cada $10 \mathrm{mS}$, por un lapso de 3 segundos, estos datos son almacenados temporalmente en el microcontrolador del sistema, luego de estos 3 segundos, el sistema calcula el punto de inflexión de la gráfica resultante, y calcula los valores de $L$ y $T$ que se muestran en la Figura 5, (OGATA, 2010).

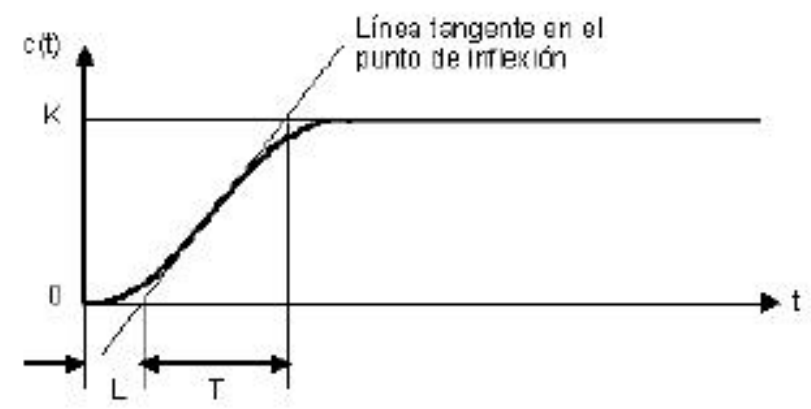

Figura 5. respuesta de un sistema de primer orden, parámetros requeridos para la sintonización por Ziegers-Nichols.

Fuente: OGATA, K. (2010). INGENIERIA DE CONTROL MODERNA $5^{a}$ ED, p. 568.

El controlador propuesto es de la forma:

$$
C=K_{P}\left(1+T_{d} s+\frac{1}{T_{i} s}\right)
$$

Con:

$$
K_{P}=1.2 \frac{T}{L} ; T_{d}=0.5 L ; T_{i}=2 L
$$

La sintonización busca el control de la velocidad de cada uno de los motores, de modo que cualquier algoritmo de control de seguimiento de trayectorias debe hacer llegar al sistema las referencias en términos de la velocidad, en una escala de 0 a 100, donde 0 equivale a una velocidad deseada de 0 en ese motor, y 100 corresponde a una velocidad deseada del $100 \%$ de la velocidad lograda por el motor. Una vez sintonizados, las señales de control son enviadas a una etapa de potencia que cuenta con 3 opciones de amplificación, para trabajar con motores a 12,24 y 36 voltios.

\section{Resultados}

Las pruebas de verificación de captura y transmisión de datos vitales se realizaron con una muestra de 5 sujetos sanos, sin enfermedades cardíacas o respiratorias, de 18 a 25 años, a esta población le fueron tomados datos por 2 minutos, mientras los sujetos se encontraban en reposo, relajados y en ayunas.

Las características de los participantes en el estudio se presentan en la Tabla 1.

Tabla 1.

Datos básicos de los participantes.

\begin{tabular}{ccccc}
\hline Sujeto & Sexo(M/F) & Edad & Peso(Kg) & Estatura(m) \\
\hline 1 & M & 18 & 65 & 1,65 \\
\hline 2 & M & 22 & 70 & 1,63 \\
\hline 3 & M & 19 & 75 & 1,81 \\
\hline 4 & F & 20 & 60 & 1,60 \\
\hline 5 & F & 19 & 55 & 1,55 \\
\hline
\end{tabular}

Fuente: elaboración propia.

Los resultados de la toma de datos en esta etapa del protocolo de medición se presentan en la Tabla 2.

Tabla 2.

Resultados de la prueba de funcionamiento de los sistemas de adquisición de datos.

\begin{tabular}{cccc}
\hline Sujeto & $\begin{array}{c}\text { Frec. Cardiaca } \\
(\mathrm{lpm})\end{array}$ & $\begin{array}{c}\text { Frec. Respiratoria } \\
(\mathrm{rpm})\end{array}$ & Temp. $\left({ }^{\circ} \mathrm{C}\right)$ \\
\hline 1 & 73 & 16 & 36 \\
\hline 2 & 67 & 18 & 36 \\
\hline 3 & 75 & 18 & 37 \\
\hline 4 & 69 & 17 & 36 \\
\hline 5 & 73 & 18 & 37 \\
\hline
\end{tabular}

Para la prueba de verificación de funcionamiento de la detección de alertas de alteraciones en las señales vitales se pidió a 2 sujetos sanos, sin enfermedades cardíacas o respiratorias (Tabla 3) con registros de actividad física de -por lo menos- 3 veces a la semana, para la verificación realizaran actividad física durante media hora y luego procedieran con el protocolo de toma de datos vitales. Las muestras se repitieron durante 5 días consecutivos. En el $95 \%$ de los casos el sistema alertaba por la alta frecuencia cardíaca al sistema de visualización computacional. 
Tabla 3.

Características de los participantes de las pruebas de detección de alertas fisiológicas

\begin{tabular}{ccccc}
\hline Sujeto & Sexo(M/F) & Edad & Peso(Kg) & Estatura(m) \\
\hline 6 & $\mathrm{M}$ & 19 & 68 & 1,74 \\
\hline 7 & $\mathrm{~F}$ & 20 & 55 & 1,50 \\
\hline Fuente: elaboración de los autores. & &
\end{tabular}

Los datos previos y posteriores a la actividad física se presentan en la Tabla 4.

Tabla 4.

Valores de las variables fisiológicas medidas antes (parte superior) y después (parte inferior) de la prueba de alertas fisiológicas.

\begin{tabular}{cccc}
\hline Sujeto & $\begin{array}{c}\text { Frec. Cardiaca } \\
(\mathrm{lpm})\end{array}$ & $\begin{array}{r}\text { Frec. Respiratoria } \\
(\mathrm{rpm})\end{array}$ & Temp. $\left({ }^{\circ} \mathrm{C}\right)$ \\
\hline 6 & 62 & 16 & 36 \\
\hline 7 & 60 & 17 & 36 \\
\hline 6 & 110 & 35 & 39 \\
\hline 7 & 120 & 37 & 38 \\
\hline Fuente: elaboración propia. & &
\end{tabular}

Después de la actividad física, las 3 alertas esperadas se alteraron, confirmando así el adecuado funcionamiento de los sistemas de adquisición de datos.

Además de los datos obtenidos, podemos decir que en estado de reposo los sujetos que realizan actividad física de manera regular, presentan frecuencias cardíacas notablemente más bajas, que quiénes no realizan actividad física de forma cotidiana.

La verificación de confiabilidad del sistema en la transmisión y toma de datos se realizó con un sujeto distinto cada día. Durante 5 días, los participantes fueron monitoreados durante 2 horas, mientras se encontraban en reposo. Para cada uno de los casos, las señales fueron enviadas al computador sin desincronización superior al $2 \%$, el sistema utiliza el dato de control para la re-sincronización de los datos en los casos en los que la trama hubiera fallado, este proceso se efectuó de forma automática.

Las pruebas de comunicación se realizaron con la especificación ZigBee, para establecer comunicaciones seguras y de bajo consumo, la especificación ZigBee permite velocidades de hasta 256.000 baudios en su versión comercial, que si bien puede ser más baja que algunas alternativas similares en el mercado, excede ampliamente los requerimientos del sistema diseñado, comparándolo con Bluetooth, que es su principal competencia, el ZigBee aprovecha mejor los recursos, de hecho el Bluetooth consume $25 \%$ más de corriente cuando está en modo de transmisión vs. ZigBee, y la diferencia alcanza un punto crítico cuando los sistemas se encuentran en reposo, ya que el ZigBee ahorra hasta $98.5 \%$ de corriente en este modo $(0,2$ $\mathrm{mA}$ en BlueTooth vs $3 \mu \mathrm{A}$ en ZigBee) (XBee, 2015).

Para verificar el funcionamiento del movimiento dinámico de la silla, se utilizaron las trayectorias generadas por un software especializado (Cifuentes Bernal, Plaza Torres, \& Castillo, 2015) que se basa en la información de un mapa conocido y aplicando algoritmos de inteligencia artificial calcula la ruta más rápida y segura entre un punto y otro.

El sistema sigue la trayectoria y llega hasta un punto cercano al deseado, pero debido a que este módulo funciona como un sistema en lazo abierto no es posible establecer un error.

\section{Conclusiones}

En la tarjeta de procesamiento central, la tecnología de 8 bits pareció quedarse corta en los estados avanzados de la propuesta, por lo que se propone un nuevo desarrollo con tecnologías de 16 bits.

En la evolución de propuesta se encontró que el manejo de tecnologías superiores, como las de 32 bits, incrementarían los costos de una posible producción en masa y dificultan el manejo de recursos de forma óptima; de hecho, la mayoría de la información podía ser tratada en unidades funcionales de un único byte, por lo que las velocidades y capacidades de procesamiento de las herramientas de nivel superior, (que si bien a simple vista parecen una mejor alternativa para esta clase de diseños) fueron descartadas por el equipo de desarrollo al considerarse subutilizadas e ir en contra de la filosofía del equipo: la optimización de los recursos del sistema.

Por otra parte, la creación de una tarjeta propia de desarrollo, en lugar de implementar una tarjeta de desarrollo comercial aumenta la versatilidad en el diseño, los recursos de hardware pueden ser empleados en su totalidad y no existen mayores limitantes en la distribución de los componentes discretos, de forma que se simplifica la creación de sistemas modulares. Sin embargo, es necesario tener muy presentes las separaciones máximas recomendadas de los distintos componentes, para evitar interacciones negativas entre las diferentes señales que convergen en la tarjeta, minimizar los efectos de capacitancias parásitas, y garantizar un adecuado muestreo de 
las señales, y la adecuada comunicación con el sistema computacional.

Además, los consumos de potencia pueden ser controlados de mejor forma, el sistema de hecho permite expansión y mejora, por esto el diseño propio se toma como la mejor alternativa para este proyecto y los de naturaleza similar.

Los módulos generados, fruto del proyecto, se probaron y se encontraron posibilidades de mejora. Se pretende reajustar el módulo de seguimiento de trayectorias como un sistema en lazo cerrado, capaz de medir un error de posición y llevarlo al mínimo posible. Además de eso se espera contrastar las medidas del sistema de medición de señales con equipos comerciales para obtener mayor confiabilidad a nuestras mediciones, y al mismo tiempo conseguir un mayor grado de autonomía que logre convertirlo en un módulo independiente que se adapte a diversos tipos de sistemas.

El trabajo modular logró satisfacer las necesidades planteadas en el diseño preliminar por eso esperamos que en trabajos futuros puedan agregarse otros módulos que alcancen nuevos objetivos (Microchip, 2006).

\section{Referencias}

Ahmad, S., Siddique, N. H., \& Tokhi, M. O. (2011). A Modular Fuzzy Control Approach for Two-Wheeled Wheelchair. Intell Robot Syst, 64, 401-426.

AL-Rousan, M., \& Assaleh, K. (2011). A wavelet- and neural network-based voice system for a smart wheelchair control. Journal of the Franklin Institute, 348, 90-100.

Cifuentes Bernal, A. M., Plaza Torres, M., \& Castillo, R. (2015). Diseño de sistemas de apoyo para silla de ruedas inteligentes empleadas por personas con problemas de movilidad debidas a lesiones de sexto nivel cervical. Tesis de Maestría, UMNG, Bogotá.

Durán Acevedo, C., \& Jaimes Mogollón, A. (2013). Optimización y clasificación de señales EMG a través de métodos de reconocimiento de patrones. ITECKNE, 67 - 76.

Fehr, L., Langbein, W. E., \& Skaar, S. B. (2000, May/June). Adequacy of power wheelchair control interfaces for persons with severe disabilities: A clinical survey. Journal of Rehabilitation Research and Development, 37(3), 353-360.

Jiménez Moreno, R., Espinosa Valcárcel, F. A., \& Amaya Hurtado, D. (2013). Teleoperated systems: a perspective on telesurgery applications. Revista Ingeniería Biomédica, 30-41.
Leishman, F., Horn, O., \& Bourhis, G. (2010). Smart wheelchair control through a deictic approach. Robotics and Autonomous Systems, 58.

Microchip. (2006). PIC18F2455/2550/4455/4550 Datasheet.

Ministerio de Asuntos Internos y Comunicaciones Oficina de Estadísticas. (1996). Statistics Japan. Retrieved 4 15, 2016, from http://mww.stat.go.jp/data/jinsui/pdf/201102.pdf

Noda, Y., Kawaguchi, A., \& Terashima, K. (2010). A Mechatronics Vision for Smart Wheelchairs. In A. Barrera (Ed.), Mobile Robots Navigation (pp. 609-628). InTech.

OGATA, K. (2010). Ingeniería de Control Moderna $5^{a}$ ED. Madrid - España: PRENTICE-HALL

Panam Post. (2015, 04 20). Retrieved 04 15, 2016, from http:// es.panampost.com/belen-marty/2015/04/20/envejecimiento-de-la-poblacion-frena-nuevos-emprendimientos-en-canada/

Plaza, M., Cifuentes Bernal, A. M., \& Aperador Chaparro, W. A. (2013). Colombia Patent No. 13-217147-00000-0000.

Statistics Canada. (2015, 11 27). Retrieved 04 15, 2016, from http:// www12.statcan.gc.ca/census-recensement/2011/dp-pd/ prof/details/page.cfm?Lang $=\mathrm{E} \&$ Geo1 $=$ PR\&Code $1=01 \&-$ Geo2=PR\&Code2 $=01 \&$ Data $=$ Count $\&$ SearchText $=$ Canada\& SearchType $=$ Begins $\&$ SearchPR $=01 \& B 1=$ All \&Custom $=\& T A-$ $\mathrm{BID}=1$

XBee. (2015). Retrieved from Que es Xbee?: http://xbee.cl/que-esxbee/ 


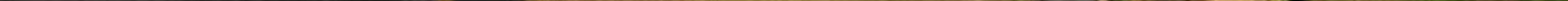

